\title{
Video Article \\ A Pipette-Tip Based Method for Seeding Cells to Droplet Microfluidic Platforms
}

\author{
Nidhi Sinha ${ }^{{ }^{1}}$, Nikita Subedi ${ }^{* 1}$, Florian Wimmers ${ }^{{ }^{2}}$, Melf Soennichsen ${ }^{1}$, Jurjen Tel $^{1}$ \\ ${ }^{1}$ Department of Biomedical Engineering and Institute for Complex Molecular Systems, Laboratory of Immunoengineering, Eindhoven University of Technology \\ ${ }^{2}$ Institute for Immunity, Transplantation, and Infection, Beckman Center, Stanford University \\ "These authors contributed equally
}

Correspondence to: Jurjen Tel at j.tel@tue.nl

URL: https://www.jove.com/video/57848

DOI: doi: $10.3791 / 57848$

Keywords: Engineering, Issue 144, Droplets, Microfluidics, Tip Loading, Poisson Distribution, Cell Encapsulation, Cell-Pairing, Cellular Interactions

Date Published: 2/11/2019

Citation: Sinha, N., Subedi, N., Wimmers, F., Soennichsen, M., Tel, J. A Pipette-Tip Based Method for Seeding Cells to Droplet Microfluidic Platforms. J. Vis. Exp. (144), e57848, doi:10.3791/57848 (2019).

\section{Abstract}

Amongst various microfluidic platform designs frequently used for cellular analysis, droplet-microfluidics provides a robust tool for isolating and analyzing cells at the single-cell level by eliminating the influence of external factors on the cellular microenvironment. Encapsulation of cells in droplets is dictated by the Poisson distribution as a function of the number of cells present in each droplet and the average number of cells per volume of droplet. Primary cells, especially immune cells, or clinical specimens can be scarce and loss-less encapsulation of cells remains challenging. In this paper, we present a new methodology that uses pipette-tips to load cells to droplet-based microfluidic devices without the significant loss of cells. With various cell types, we demonstrate efficient cell encapsulation in droplets that closely corresponds to the encapsulation efficiency predicted by the Poisson distribution. Our method ensures loss-less loading of cells to microfluidic platforms and can be easily adapted for downstream single cell analysis, e.g., to decode cellular interactions between different cell types.

\section{Video Link}

The video component of this article can be found at https://www.jove.com/video/57848/

\section{Introduction}

In recent years, the use of microfluidics as a robust and versatile platform for cellular analysis at the single cell level has rapidly increased ${ }^{1}$. These platforms provide high-throughput screening of single cells and biological molecules with high precision and sensitivity using very small sample volume ${ }^{2,3,4}$. Among different types of microfluidic designs, droplet-based platforms enable high-throughput analysis of single cells by isolating them in an aqueous phase droplet surrounded by an immiscible phase that allows precise and accurate control over the cellular microenvironment ${ }^{5,6}$. Droplet-based microfluidics gives the flexibility to isolate single or multiple-cells in, both, aqueous and hydrogel droplets and is valuable in probing complex cellular behavior, such as protein secretion or cellular interactions ${ }^{7,8,9}$. Signaling and cross-talk amongst immune cells can be influenced by interactions with other cells in the microenvironment ${ }^{10}$. Isolation of single cells in droplets provides an effective noisefree analytical laboratory, free from the influence of external environmental factors for more efficient and accurate results ${ }^{11,12}$. Modifying the design of a droplet-microfluidic platform with multiple inlets allows the encapsulation of multiple cell types to study cellular interactions via cellpairing ${ }^{12,13}$.

The process of encapsulation of cells in droplets is random and the rate of encapsulation of cells can be statistically determined using the formula for the Poisson distribution ${ }^{14,15}$. This rate of encapsulation can be estimated by considering the average rate of the arrival of cells at the droplet junction and assuming that the arrival of each cell is independent from the arrival of other cells ${ }^{16}$. Even though independent cell arrival cannot be guaranteed, in cases of sparsely distributed cells, the assumption of independence can be considered and the probability of a droplet containing one or more cells can be predicted as a function of the number of cells present in each droplet and the average number of cells per droplet $^{16,17}$. Since this estimation of cellular encapsulation in droplets is dependent on the number of cells present in each droplet, one can suggest that increasing the concentration of the cells at the inlet will increase the average number of cells present in each droplet ${ }^{16}$. Therefore, to ensure single cell encapsulation, the cell concentrations must be reduced but this often leads to a large number of empty droplets ${ }^{18}$.

Loss of cells during loading by either attachment, sedimentation, and/or clumping in the syringe, tubing, or production device is a common drawback responsible for the deviation of actual encapsulation values from the predicted encapsulation values ${ }^{19}$. This problem gets further exaggerated when seeding rare immune cells or clinical specimens as they are already scarce in population and the encapsulation of only a few cells, much lower than expected, does not provide sufficient data for experimental analysis. Plasmacytoid dendritic cells (pDCs) are a rare subset of immune cells that only constitutes approximately 0.2 - 0.6 percent of the entire white blood cell population ${ }^{20}$. These cells secrete massive amounts of type I interferons upon activation and thereby play a critical role in immune responses ${ }^{21}$. When studying the cellular behavior of such rare cells in droplets, it is imperative to prevent cell loss during cell seeding and encapsulation ${ }^{22}$. There are several design related developments that have ensured the encapsulation of single cells in droplets using active encapsulation methods that utilize different physical forces such as 
acoustic or electrical forces for generation of droplets containing single-cells ${ }^{23,24}$. However, these methods have their own limitations in terms of droplet production ${ }^{16}$.

In this study, we established a robust and straightforward method that circumvents the shortcomings of traditional methods for loading single or multiple cells to microfluidic devices. Our method, inspired by Rho et al., utilizes differently-sized pipette tips for seeding small volumes of rare immune cells to droplet microfluidic platforms without significant sample loss and yielded results that are coherent with theoretical predictions ${ }^{25}$. This methodology can be easily and successfully adapted for several applications involving droplet-based microfluidics and applied for a wide variety of cell types or even microparticles.

\section{3-Inlet Polydimethylsiloxane (PDMS) Device Fabrication}

1. Measure $40 \mathrm{~g}$ of PDMS base in a conditioning mixer cup and add $4 \mathrm{~g}$ of PDMS curing agent to the base reagent in the cup, carefully, using a dropper.

2. Place the cup in the holder of the conditioning mixer and measure the total weight of the cup with the holder. Set the value of the centrifuge balance weight on the conditioning mixer accordingly.

3. Mix the base and curing agent in the conditioning mixer at 2,000 rpm for $2 \mathrm{~min}$ followed by de-foaming at 2,000 rpm for $2 \mathrm{~min}$.

4. Prepare an aluminum boat, with a diameter approximately the same size as that of a $100 \mathrm{~mm}$ silicon wafer. Place the silicon wafer, fabricated for the replica molding process, in the aluminum boat and put this setup in a Petri dish (diameter $=120 \mathrm{~mm}$, height $=20 \mathrm{~mm}$ ). NOTE: The size of the Petri dish depends on the size of the silicon wafer.

5. Remove the cup from the holder and pour the pre-cured PDMS mixture (contents of the cup), carefully, on the silicon wafer.

6. Place the Petri dish, containing the silicon wafer with the pre-cured PDMS mixture, in a desiccator for about 20 min to remove all the air bubbles.

7. Remove the Petri dish after 20 min and check for any remaining air bubbles that can be removed.

8. Place the Petri dish in an oven, set at $65^{\circ} \mathrm{C}$, for at least $3 \mathrm{~h}$.

9. Remove the Petri dish from the oven after $3 \mathrm{~h}$ and carefully peel the cured PDMS from the silicon wafer.

10. Cut PDMS devices along the cut lines, using a knife or a scalpel. Punch holes at the inlets and outlet of each device using a $1.2 \mathrm{~mm}$ puncher. Clean each PDMS device with scotch tape to remove any dust or residual pieces of PDMS.

1. Optionally, blow with nitrogen to remove residual PDMS pieces.

11. Prepare glass slides by cleaning them with soap-water, followed by isopropanol and dry with nitrogen.

12. Bond a clean PDMS device with a clean glass slide in a plasma asher to close the flow lines. Use the following settings: Power: $50 \mathrm{~W}$, Time: $45 \mathrm{~s}$, Bleed delay time: $2 \mathrm{~s}$, Process gas: Gas 1 (Air), Vent: Both valves, Restricted vent time: $60 \mathrm{~s}$, Pump spin down time: $10 \mathrm{~s}$, Vent hold time: $0 \mathrm{~s}$, Gas shutoff time: $1 \mathrm{~s}$, Turbo pumping enabled: 0 . Disconnect all the other gas lines.

NOTE: The settings used for the plasma asher can vary according to the brand of the plasma asher used.

13. Prepare the silane solution by adding $50 \mu \mathrm{L}$ of silane $(1 \mathrm{H}, 1 \mathrm{H}, 2 \mathrm{H}, 2 \mathrm{H}$-Perfluorooctyltriethoxysilane) to $950 \mu \mathrm{L}$ of fluorinated oil. NOTE: Silane is toxic. Please operate under fume hood.

14. Draw the prepared silane solution in a syringe, which is connected to a Teflon tubing.

15. Salinize the device by flushing the prepared silane solution through the outlet of the device.

16. Place the device in an oven, set at $65^{\circ} \mathrm{C}$, for $30 \mathrm{~min}$.

17. Remove the salinized device from the oven and flush excess silane out of the device with fluorinated oil.

18. Place the device back in an oven, set at $65^{\circ} \mathrm{C}$, for at least $1 \mathrm{~h}$ to complete the bonding process. Note: The Protocol can be paused here.

\section{Loss-Less Cell Encapsulation}

\section{Cell harvesting}

1. Re-suspend Jurkat T cells in Roswell Park Memorial Institute (RPMI) medium at concentrations of $1.0 \times 10^{6}$ cells $/ \mathrm{mL}, 2.0 \times 10^{6}$ cells $/ \mathrm{mL}$, $4.0 \times 10^{6}$ cells $/ \mathrm{mL}$, and $8.0 \times 10^{6}$ cells $/ \mathrm{mL} ; \mathrm{pDCs}$ in hematopoietic serum-free culture media (e.g., X-VIVO 15) at concentrations of $1.3 \times 10^{6}$ $\mathrm{cells} / \mathrm{mL}, 3.0 \times 10^{6} \mathrm{cells} / \mathrm{mL}$, and $13.0 \times 10^{6} \mathrm{cells} / \mathrm{mL}$; A549 cells in Dulbecco's Modified Eagle's Medium (DMEM) at a concentration of $1.0 \times 10^{6} \mathrm{cell} / \mathrm{s} / \mathrm{mL}$.

NOTE: The type of cells and concentration of cells can vary based on the experiment. Labelling of cells can also be done based on the experiment.

2. Tip-loading for aqueous droplet generation

1. Prepare fluorinated oil with $3 \%$ biocompatible surfactant mixture by adding $3 \mathrm{~mL}$ of surfactant to $2 \mathrm{~mL}$ of fluorinated oil. NOTE: The concentration of the surfactant added to the fluorinated oil determines the stability of the emulsion for different incubation periods. The concentration of the surfactant varies depending on the media used for specific cell-types.

2. Draw the oil phase mixture in a syringe $(1 \mathrm{~mL})$. Remove air bubbles from the syringe and connect it to a Teflon tubing of appropriate length.

3. Prepare a sample syringe by drawing biocompatible mineral oil in a syringe. Remove air bubbles and connect the syringe to a Teflon tubing of appropriate length.

4. Punch a PDMS plug with a diameter of $5 \mathrm{~mm}$ from a cured PDMS slab.

NOTE: The cured PDMS slab can be prepared using steps 1.1 to 1.9. Use a plain silicon wafer instead of a fabricated silicon wafer.

5. Punch another hole in the center of the plug with a $1 \mathrm{~mm}$ puncher.

6. Insert the plug in a $200 \mu \mathrm{L}$ pipette tip, from the larger end, such that it fits tightly. 
NOTE: Use a 1,000 $\mu \mathrm{L}$ pipette tip for larger sample volume and larger cells. For 1,000 $\mu \mathrm{L}$ pipette tip, plugs of diameter ranging between $5 \mathrm{~mm}$ and $7 \mathrm{~mm}$ can be used. With a plug of diameter $5 \mathrm{~mm}$, a sample volume of around $400 \mu \mathrm{L}$ can be aspirated in the pipette tip. If a plug of larger diameter is used $(7 \mathrm{~mm})$, more sample volume can be aspirated (around $900 \mu \mathrm{L}$ ).

7. Insert the tubing, which is connected to the syringe, in the PDMS plug, which has been inserted in the pipette tip. Push the syringe plunger slowly to fill the connected pipette tip with mineral oil. Push out all the residual air from the pipette tip.

8. Lower the pipette tip, connected to the syringe, in the sample solution and aspirate about $150 \mu \mathrm{L}$ of sample in the tip.

9. Repeat steps 2.2 .4 to 2.2 .8 to prepare a second sample syringe.

10. Carefully place all the three prepared syringes on the syringe pump.

11. Insert both the pipette tips, containing the sample, in the two inner inlets of the PDMS chip. Insert the tube containing the oil phase mixture in the outer inlet.

12. Set the value of the flow rates on the syringe pump as follows: continuous phase solution: $600 \mu \mathrm{L} / \mathrm{h}$, cell samples: $100 \mu \mathrm{L} / \mathrm{h}$, each. Enter and set the dimensions of the syringe.

NOTE: The diameter settings will vary based on the type of syringe.

13. Start the pump to flush sample solution through the inner channels of the device and oil phase through the outer channel of the device.

14. Plug in a tubing of appropriate length to the outlet to start collecting the droplets when the droplet formation is stable. The time of collection varies based on the experiment.

15. Collect droplets in a lock tube. Add $200 \mu \mathrm{L}$ of RPMI medium (without serum) on top of the collected droplets and incubate the sample. NOTE: Incubation time of the collected droplets varies based on the experiment. Droplets are collected in a lock tube when flow cytometry-based analysis or isolation is performed after retrieving the cells from droplets by breaking the emulsion. It is possible to collect the droplets in a glass chamber if the experiment requires in-droplet microscopic analysis.

\section{Emulsion breaking and cell retrieval for flow cytometric analysis}

1. Prepare $20 \% 1 \mathrm{H}, 1 \mathrm{H}, 2 \mathrm{H}, 2 \mathrm{H}$-Perfluoro-1-octanol (PFO) solution ( $\mathrm{v} / \mathrm{v}$ ) in fluorinated oil by adding $2 \mathrm{~mL}$ of PFO in $10 \mathrm{~mL}$ of flourinated oil.

2. Remove excess oil from the bottom of the collection tube, containing the droplets, using a syringe.

3. Add $100 \mu \mathrm{L}$ of $20 \%$ PFO solution to the emulsion to break the emulsion and release the encapsulated cells into the aqueous phase. Tap and mix briefly. Do not vortex at this point. Incubate for 1-2 min.

NOTE: The amount of PFO added depends on the quantity of droplets produced. Keep adding additional PFO until the oil layer is completely dissolved. Keep in mind that PFO is toxic for the cells and that too high PFO concentrations or too long incubation in PFO might lead to increased cell death.

4. Spin the solution shortly at the lowest possible rcf for $30 \mathrm{~s}$.

5. Prepare $100 \mathrm{~mL}$ cold Phosphate-Buffered Saline (PBS) solution supplemented with $2 \%$ Fetal Calf Serum (FCS) (2 mL of FCS in $98 \mathrm{~mL}$ of PBS).

6. Pipette $550 \mu \mathrm{L}$ of the aqueous fraction, immediately after centrifugation and transfer it to a new lock tube containing $500 \mu \mathrm{L}$ of cold PBS solution supplemented with $2 \%$ FCS, as prepared in step 2.3.5. Let any residual oil sink to the bottom of the new lock tube.

7. Aspirate $950 \mu \mathrm{L}$ of the aqueous phase containing the cells from this lock tube, carefully, without aspirating any residual oil and transfer the solution to a new lock tube.

8. Spin down the cells in the new lock tube for $10 \mathrm{~min}$.

9. Re-suspend the cells in $300 \mu \mathrm{L}$ of cold PBS solution supplemented with $2 \%$ FCS, as prepared in step 2.3.5.

NOTE: The cells can be also re-suspended in any other suitable solution such as media depending on the experiment.Stain the cells, based on the experiment, for analysis using flow cytometry.

\section{Cell Pairing}

\section{Cell harvesting and staining}

1. Count Jurkat T cells, from culture flask, and spin down the cells at $1,500 \mathrm{rpm}$ for $5 \mathrm{~min}$.

2. Remove the supernatant and re-suspend $1.0 \times 10^{6}$ cells in $1 \mathrm{~mL}$ PBS to get a concentration of $1.0 \times 10^{6}$ cells $/ \mathrm{mL}$. The amount of PBS added depends on the cell count.

3. Repeat steps 3.1.1 to 3.1.2 to prepare a second sample of Jurkat T-cells with the same cell concentration.

4. Wash both samples twice with $1 \mathrm{~mL}$ of PBS at $1,500 \mathrm{rpm}$ for $5 \mathrm{~min}$.

5. Re-suspend one cell sample with $1.25 \mu \mathrm{M}$ carboxyfluorescein succinimidyl ester (CFSE) dye and the other cell sample with $1.25 \mu \mathrm{M}$ far red dye or $1.25 \mu \mathrm{M}$ cell proliferation dye at a cell concentration of $1.0 \times 10^{6}$ cells $/ \mathrm{mL}$. The total staining solution is $1 \mathrm{~mL}$ for $1.0 \times 10^{6}$ cells. NOTE: Cells can be labelled with different dyes depending on the filters available in the flow cytometer or in the fluorescence microscope.

6. Incubate the cell samples with dyes for $10 \mathrm{~min}$ at $37^{\circ} \mathrm{C}$.

7. Stop the staining reaction by adding $1 \mathrm{~mL}$ of ice cold FCS after $10 \mathrm{~min}$

8. Wash the cell samples twice with $1 \mathrm{~mL}$ of PBS at $1,500 \mathrm{rpm}$ for $5 \mathrm{~min}$.

9. Re-suspend the cell samples in RPMI media at a concentration of $10.0 \times 10^{6}$ cells $/ \mathrm{mL}$, for each color.

\section{Tip-loading for production of agarose hydrogel droplets for cell pairing}

Note: For cell pairing using agarose hydrogel droplets, maintain the temperature of the system between $27^{\circ} \mathrm{C}$ and $37^{\circ} \mathrm{C}$ throughout the droplet generation and collection process to prevent gelling of the hydrogels and to warrant cellular viability ${ }^{9}$.

1. Dissolve ultra-low gelling temperature agarose by heating it up to $75{ }^{\circ} \mathrm{C}$ in PBS at a concentration of $4 \%$ (w/v) and stir the mixture for $20 \mathrm{~min}$.

2. Mix the agarose solution with labelled Jurkat T cells to yield an agarose concentration of $2 \%(w / v)$. Repeat this for the other sample with differently labelled cells.

3. Prepare fluorinated oil with $2 \%$ surfactant mixture by adding $20 \mathrm{~mL}$ of surfactant to $30 \mathrm{~mL}$ of fluorinated oil (oil phase mixture).

4. Follow the steps $2.2 .2-2.2 .14$. 
NOTE: Because of the viscous nature of low melting agarose and to ensure stable droplet production, set the value of the flow rates on the syringe pumps as follows: oil phase mixture: $2,000 \mu \mathrm{L} / \mathrm{h}$, cell samples: $200 \mu \mathrm{L} / \mathrm{h}$. Enter and set the dimensions of the syringe.

5. Collect the droplets in a lock tube and incubate the droplets at $4{ }^{\circ} \mathrm{C}$ for $60 \mathrm{~min}$.

\section{Emulsion breaking and agarose bead retrieval for FACS analysis}

1. After incubation of droplets for $60 \mathrm{~min}$, remove excess oil from the lock tube, containing the droplets, using a syringe.

2. Add $200 \mu \mathrm{L}$ of PFO to remove the oil interphase from the droplets.

NOTE: The amount of PFO added to the tube depends on the quantity of droplets produced. Keep adding additional PFO until the oil layer is completely dissolved.

3. Wash the collected agarose beads twice with $1 \mathrm{~mL}$ of cold PBS to remove oil completely by centrifugation at $1,500 \mathrm{rpm}$ for $10 \mathrm{~min}$.

4. Analyze collected agarose beads using flow cytometry.

NOTE: It is also possible to observe the beads under a fluorescence microscope.

\section{Representative Results}

For our experiments, we used a 3-inlet PDMS based microfluidic device with the height of 25 microns (Figure 1). In this device setup, we used the outer inlet for flushing the oil with surfactant and the two inner inlets for flushing the aqueous phases with cell suspensions or media. After generation and collection, the droplets are incubated for a couple of hours off chip before downstream analysis using flow cytometry. During the incubation period, serum components present in the media can interact with the surfactant and cause droplets to become unstable and disintegrate. It is therefore important to add an optimized concentration of surfactant to the fluorinated oil. We tested the stability of the monodispersed droplets containing hematopoietic serum-free culture media supplemented with $2 \%$ human serum with different concentrations of surfactant in fluorinated oil. It can be inferred from Figure 2 that these monodispersed droplets are highly stable for up to 24 hours when at least $3 \%$ surfactant is added to the oil phase. Similar results were obtained with RPMI media with and without the addition of $10 \%$ FCS (data not shown). Therefore, droplet stability is highly dependent on optimal surfactant concentrations when working with different sources of culture media and serum components.

To demonstrate the encapsulation efficiency of our approach we first seeded the cells using tubing connected to syringes, which is the most conventional approach for seeding cells (Figure 3A). We harvested Jurkat T cells at different concentrations of $1.0 \times 10^{6} \mathrm{cells} / \mathrm{mL}, 2.0 \times 10^{6} \mathrm{cells} /$ $\mathrm{mL}$, and $4.0 \times 10^{6}$ cells $/ \mathrm{mL}$ and obtained an encapsulation efficiency that was lower than predicted values (Figure 3B). At 1.0x10 $0^{6} \mathrm{cells} / \mathrm{mL}$, the fraction of droplets that contained a single cell was $2.5 \%$, which did not increase even upon using higher cell concentrations. To increase the cell-loading efficiency, we modified our previous approach and mounted the tubing at half the length to an elevated tripod and loaded the cell suspension in the half that was attached to the PDMS device (Figure 4A). Using this approach, we encapsulated Jurkat T cells at different concentrations of $1.0 \times 10^{6} \mathrm{cells} / \mathrm{mL}, 2.0 \times 10^{6} \mathrm{cells} / \mathrm{mL}$, and $4.0 \times 10^{6}$ cells $/ \mathrm{mL}$, and also rare $\mathrm{pDCs}$ at different concentrations of $1.0 \times 10^{6}$ cells/ $\mathrm{mL}, 2.0 \times 10^{6} \mathrm{cells} / \mathrm{mL}$, and $12.0 \times 10^{6}$ cells $/ \mathrm{mL}$. We expected improved encapsulation rates by preventing cell sedimentation with this method. However, at all the concentrations tested, the experimental results were much lower that the predicted Poisson values (Figure 4B and Figure 4C).

Using our tip-loading approach we optimized our cell encapsulation rates to obtain experimental results coherent with the statistically predicted values (Figure 5A). For different concentrations of Jurkat T cells, the obtained encapsulation efficiency matched our calculated values at all concentrations (Figure 5B). Remarkably, even with adherent cells like A549 tumor cells, which tend to clump, we observed a slightly improved encapsulation efficiency at a cellular concentration of $1.0 \times 10^{6}$ cells $/ \mathrm{mL}$ (Figure $5 \mathrm{C}$ ). We also assessed the efficacy of our system to encapsulate less available and scarce pDCs at different cell concentrations of $1.0 \times 10^{6}$ cells $/ \mathrm{mL}, 3.0 \times 10^{6} \mathrm{cells} / \mathrm{mL}$, and $13.0 \times 10^{6} \mathrm{cells} / \mathrm{mL}$ (Figure $5 \mathrm{D}$ ). To facilitate the loading of possibly larger volumes exceeding $200 \mu \mathrm{L}$, e.g., when working with cell lines or more abundant primary immune cells, we also investigated the cell encapsulation efficiency by using $1000 \mu \mathrm{L}$ tips (blue). We demonstrated that these $1,000 \mu \mathrm{L}$ tips gave a similar encapsulation efficiency in comparison to the $200 \mu \mathrm{L}$ tips (yellow) (Figure 5E).

Dependent on the chip design and research question at hand, our tip loading technique can be used to load cells through either one inlet, for probing into cellular heterogeneity, or multiple inlets in parallel, for decoding cellular interactions. We compared the loading of Jurkat $\mathrm{T}$ cells (at a concentration of $10.0 \times 10^{6}$ cells $/ \mathrm{mL}$ ) from one inlet to two differently labelled populations of Jurkat T cells (at a combined concentration of $10.0 \times 10^{6} \mathrm{cells} / \mathrm{mL}$ ) from two inlets (Figure 6A and Figure 6B). During encapsulation, the droplets were generated using ultra-low gelling temperature agarose and gelled after production to form agarose hydrogel beads that allowed subsequent downstream analysis via microscopy and flow cytometry (Figure 6C and Figure 6D). Microscopic analysis revealed that cell pairing was achieved at different combinations indicating for high throughput cell pairing (Figure 6C). Furthermore, analysis of the same population of hydrogel beads by flow cytometry revealed that beads without cells could be separated from beads with cells based on the distinct forward (FSC, size) and sideward (SSC, granularity) scatter pattern (Figure 6D). Gating on the population of beads without cells confirmed a lack of cell encapsulation by the absence of fluorescent signals. Additionally, gating on the bead population with cells revealed the existence of multiple sub-populations indicative for the encapsulation of differently labeled Jurkat T cells. Our results demonstrate that efficient cell pairing can be achieved, based on both microscopic and flow cytometric analysis, and showed a slightly increased encapsulation efficiency compared to the Poisson prediction (Figure 6E). 


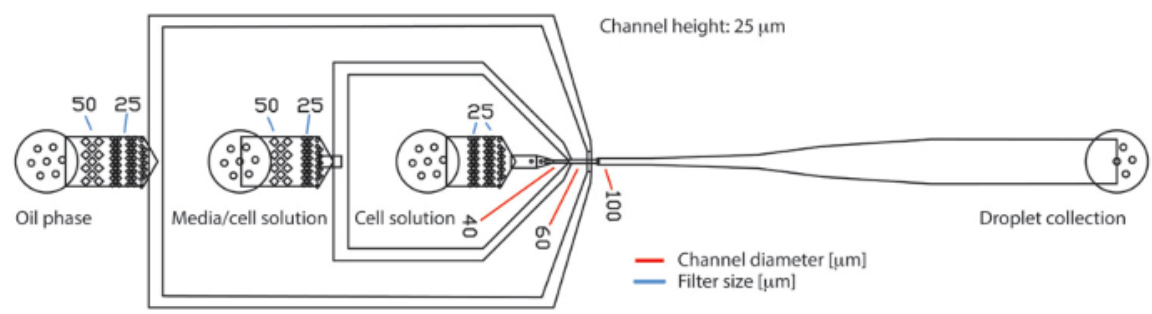

Figure 1. PDMS based droplet microfluidic device with three inlets and one outlet. The device consists of three inlets for continuous oil phase, cell culture media, and cell suspension, respectively. The generated droplets are collected at the outlet. The samples flow laminarly to the flow-focusing junction where they are encapsulated in droplets. At the inlets, filter structures hold large particles like protein or cell aggregates back. The diameter of the gaps in the filter structure are indicated by blue lines. The diameter of the channels at the production nozzle are indicated by red lines. The channel height on the entire chip was $25 \mu \mathrm{m}$. Please click here to view a larger version of this figure.
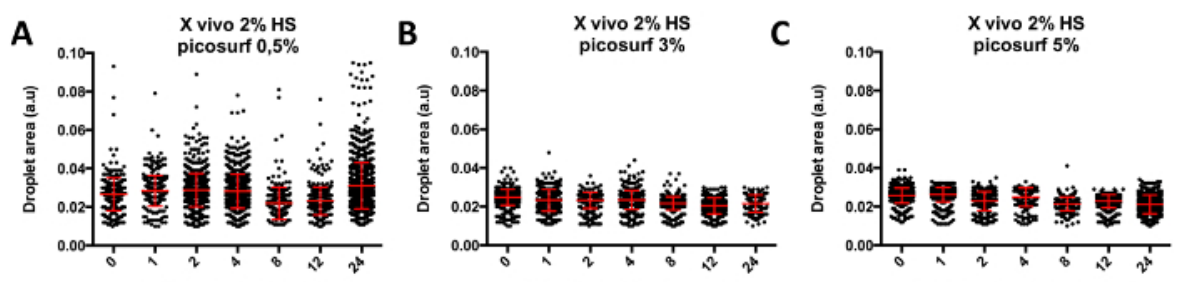

Hours after droplet production

Hours after droplet production

Hours after droplet production

Figure 2. Droplet stability over 24 hours. The graphs show the area of droplets containing hematopoietic serum-free culture media $+2 \%$ human serum, over time for three different concentrations of surfactant A) $0.5 \%$ B) $3 \%$ C) $5 \%$. Please click here to view a larger version of this figure.
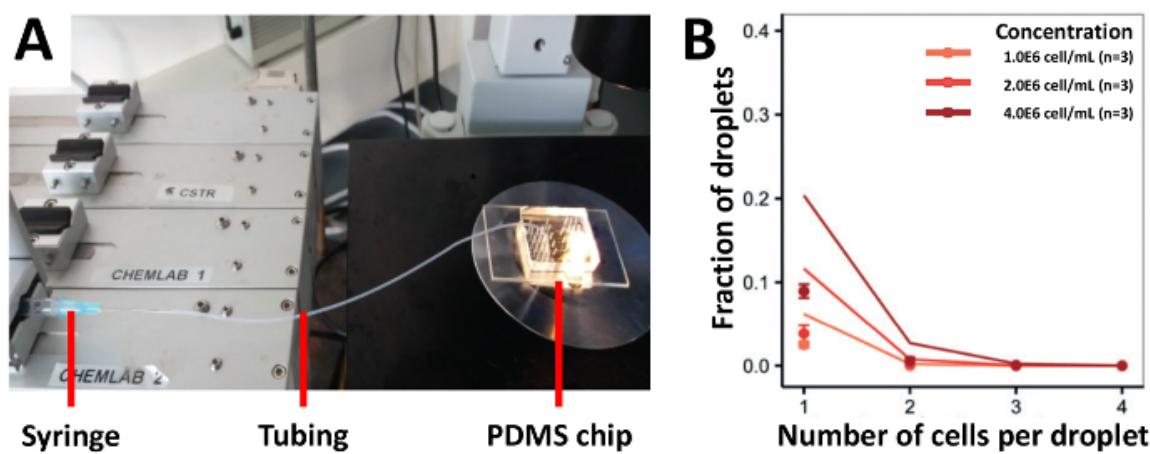

Figure 3. Tubing based cell loading approach. Jurkat-T cells are loaded at different concentrations to the device using a syringe connected to tubing. A) The illustration shows the experimental setup B) The cell encapsulation rate as determined by light microscopy. Dots: experimentally determined values; Closed lines: Poisson distribution. Error bar represents standard error of mean. Please click here to view a larger version of this figure. 

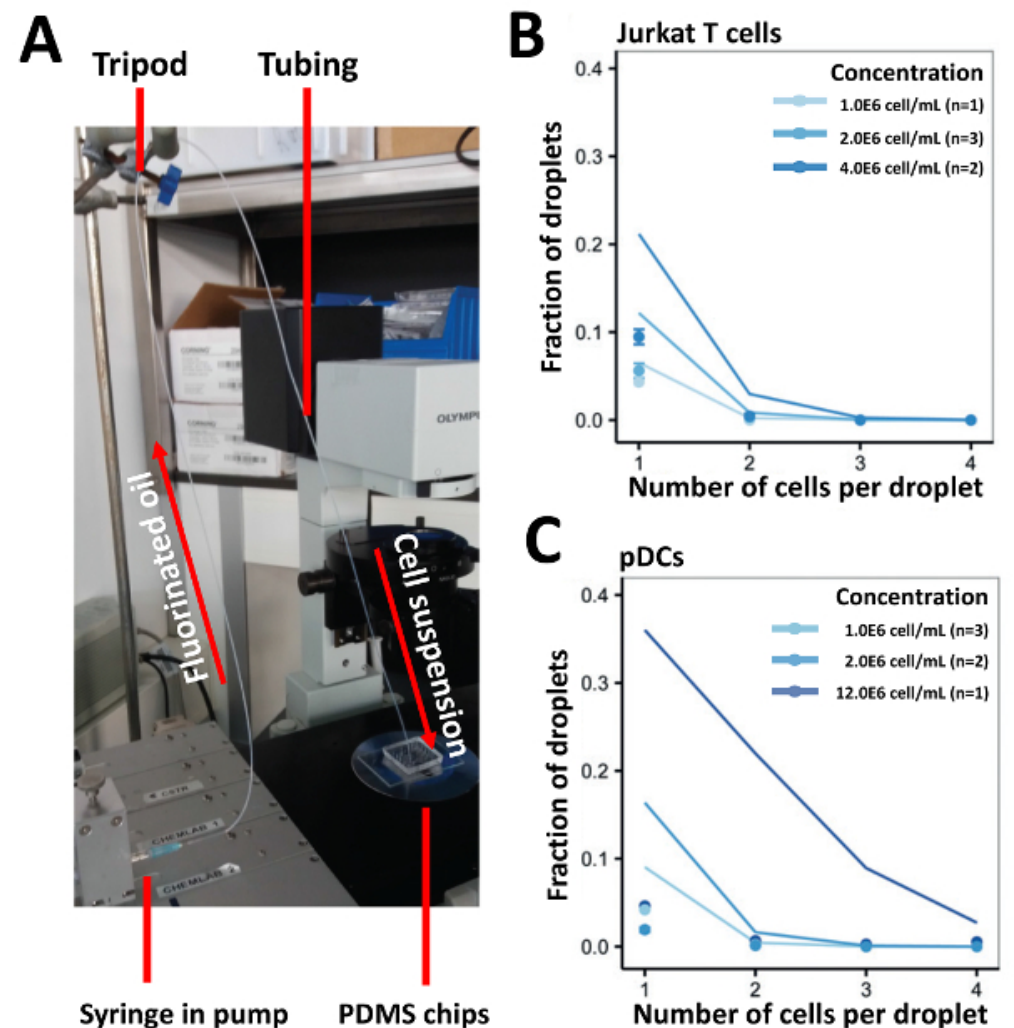

Figure 4. Encapsulation of various cell types at different concentrations using a vertical tube loading approach. Jurkat $T$ cells and pDCs (of different concentrations) were encapsulated to determine the efficiency of cell encapsulation. A) The illustration shows the experimental setup for the vertical tube loading approach. B) The graph shows the encapsulation efficiency of Jurkat T cells. C) The graph shows the encapsulation efficiency of pDCs. Dots: experimentally determined values; Closed lines: Poisson distribution. Error bar represents standard error of mean. Please click here to view a larger version of this figure. 
A

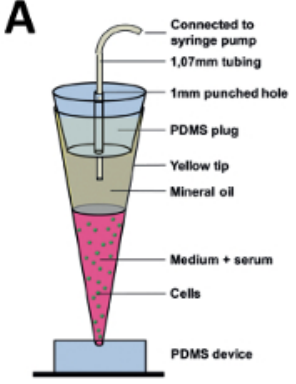

C A549 cells

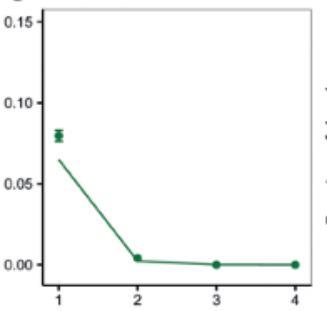

Number of cells per droplet

E Jurkat T cells

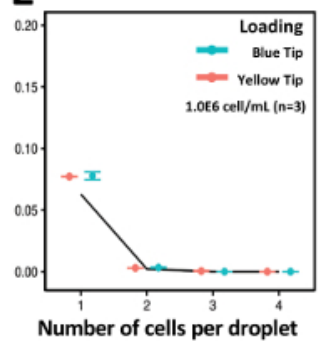

B Jurkat T cells

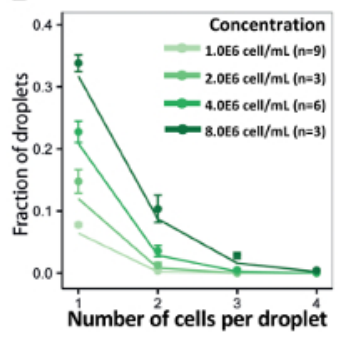

D pDCs

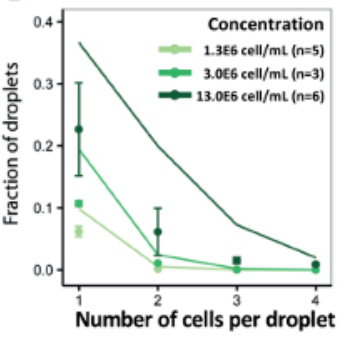

Figure 5. Tip loading approach to encapsulate different cells types. A) Schematic illustration of the tip loading technique. B) The graph shows the Encapsulation efficiency of Jurkat cells. C) The graph shows the encapsulation efficiency of A549 cells. D) The graph shows the encapsulation efficiency of pDCs. E) The graph shows the encapsulation efficiency of Jurkat T cells using $200 \mu \mathrm{L}$ pipette tips (yellow) and 1,000 $\mu \mathrm{L}$ pipette tips (blue). Dots: experimentally determined values; Closed lines: Poisson distribution. Error bar represents standard error of mean. Please click here to view a larger version of this figure. 

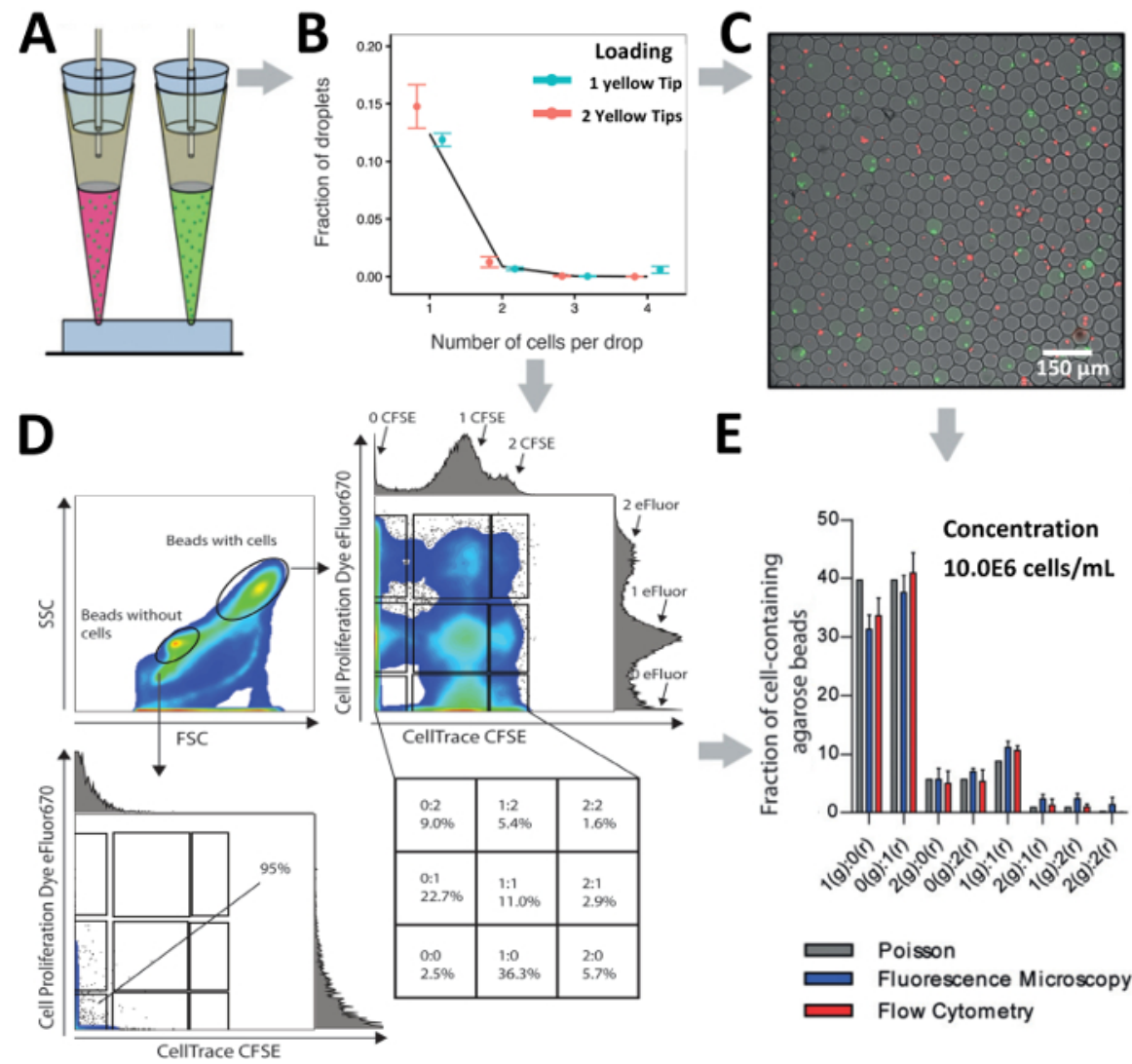

$\mathbf{E}$

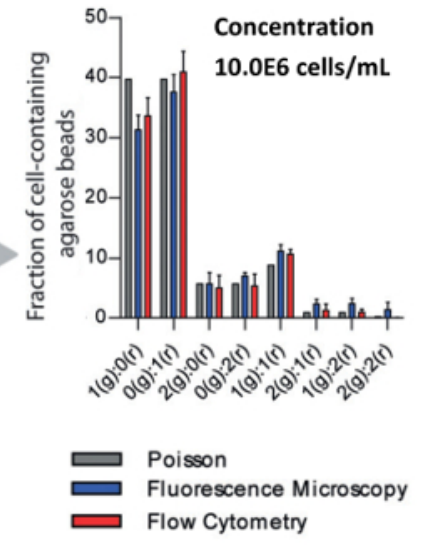

Figure 6. Cell pairing in droplets. A) Schematic illustration of the tip loading approach for pairing distinct cells from 2 inlets in droplets. B) The graph shows the encapsulation of Jurkat T cells using one inlet or two inlets in parallel. The cell concentration for one tip is $2.0 \times 10^{6} \mathrm{cells} / \mathrm{mL}$ and the cell concentration for two tips both $1.0 \times 10^{6}$ cells $/ \mathrm{mL}$. Dots: experimentally determined values; Closed lines: Poisson distribution. C) The Fluorescence microscopic overlays of hydrogel beads and labelled Jurkat T cells. D)The graph shows the flow cytometric analysis of paired cells in agarose hydrogel beads. The plot demonstrates both forward scatter and sideward scatter. E) Comparison of cell numbers in agarose hydrogel beads as detected by fluorescence microscopy and flow cytometry. Bars: mean value; Whiskers: standard error of mean, $n \geq 4$. Error bar represents standard error of mean. Please click here to view a larger version of this figure.

\section{Discussion}

In this protocol, we have demonstrated an efficient and straightforward technique to load and encapsulate cells in droplets for high-throughput, single-cell analysis and to perform controlled cell pairing for cellular interaction studies. Furthermore, we have compared several conventional approaches to load cells to microfluidic devices and showed that our tip loading approach is a more efficient technique in comparison to other methods.

Studying clinical specimens or rare cell types scarce in number by droplet-based microfluidics possess some inherent challenges. Like we have also demonstrated, cells tend to sediment in syringes and surface of the tubing, thereby, preventing cellular encapsulation to conform to the predicted values. To evade this problem, some groups use stirring bars in the syringes. However, when using rare and limited cell populations, the total cell volume is also limited, thereby, limiting the use of large syringes and stirring bars. Furthermore, we also replaced more commonly used tubing with Teflon coated tubing to prevent cell attachment but this method did not improve the results and if the tubing is too long, the problem of cell attachment aggravates (data not shown). Alternatively, we used a vertical tube loading approach where the cells were loaded in the tubing and not in the syringe to prevent the loss of cells in large syringe volumes. Using this technique, cells with small sample volume can be loaded, e.g., pDCs which are rare and limited. Also, the sample from the tubing is loaded to the device vertically to prevent cell sedimentation. The tubing used for cell seeding has small dimensions and can be compared to microchannels. The flow in the tubing is pressure driven and follows a parabolic velocity profile ${ }^{26}$. This implies that the maximum flow velocity is at the center of the tubing and minimum velocity is at the edges of the tubing ${ }^{27}$. When flushing a population of cells through the tubing, the velocity gradient causes the cells to be pushed towards the edges where they settle down because the velocity at the boundary is close to zero. The sedimentation or settlement of cells in the tubing, thereby, reduces the encapsulation efficiency as shown in the representative results where the experimental data did not match with the predicted model.

Another commonly adapted solution used by scientists, working with droplet microfluidics, is to increase the density of the cell culture media by addition of density matching reagents such as lodinaxol to prevent cell sedimentation in syringes ${ }^{19}$. However, density matching reagents can influence cellular behavior and adversely affect the cytokine secretion by cells (data not shown) ${ }^{28}$.

Even though several small and big modifications in conventional cell loading techniques showed slight improvements in encapsulation efficiencies, the obtained experimental results still did not match the theoretical calculations. However, with the tip loading approach we could 
the overcome the limitations of previous methods and encapsulation efficiency governed by Poisson statistics. This technique is not only advantageous for loading suspension cells but can also be applied for loading adherent cells, such as primary keratinocytes and A549 to microfluidic chips. When using abundant cell lines, for example A549, K562, etc., larger sample volume can be used. Therefore, depending on the volume of the sample, different sized pipette-tips can also be used and this simple technique can be adapted for both single-cell encapsulation and multiple cell encapsulation.

While low cell concentration is required to ensure the encapsulation of single cells in droplets, higher cells concentrations are desired to increase the average number of cells encapsulated in each droplet for studies related to cell pairing. There are several single-cell methods that have been previously described to pair immune cells on microfluidic chips or microfabricated nanowells ${ }^{29,30,31}$. In droplet microfluidics, Poisson statistics dictates that 1:1 cell pairing for two different cell types can be achieved at optimal cell concentrations. Based on the Poisson prediction, there is also a probability that droplets might contain other combinations. While 1:1 cell pairing can be desirable to study cellular interactions at single cell level and results in increased cellular understanding, multiple cell pairing also has major advantages. It allows to comprehend the influence of multiple cells of one cell type on the other cell type. Cross talk between different immune cells help to generate an effective immune response against several infections and pathogens and also adds robustness to our immune system ${ }^{32}$. As such, cellular communication can be interrogated with high precision in distinct contexts, e.g., 1:1, 2:1, 1:2, 2:2, 3:1, etc. yielding increased understanding on how single or pairs of cells control the induction of immune responses. This is particularly interesting to study for example the capacity of natural killer cells or cytotoxic T cells to serially kill their respective target cells.

As discussed, for multiple encapsulation of cells in droplets, higher cell concentrations are desired. However, when loading cells from one inlet for cell encapsulation, higher concentrations of cell sample can cause cells to aggregate at the inlet. This results in lower encapsulation rates and higher deviation from the theoretical values. To evade this problem, the cells can be loaded from two separate inlets as well. Theoretically, it would be possible to develop other microfluidic devices with multiple inlets to achieve even higher levels of cell encapsulation where an average on $x$ number of cells is warranted. In this study we investigated the encapsulation efficiency of Jurkat T cells when loaded from both one inlet and two inlets using the same total concentration and obtained similar encapsulation efficiency. This modification allows researchers to pair different cell types on chip.

While this method aids in loading cells to microfluidic devices without significant loss of cells, there are certain precautions that need to be kept in mind. When filling the syringes with mineral oil and aspirating the cell sample in pipette tips, incorporation of air bubbles should be avoided and the entire system should be air-free. It is also important to keep in mind that the mineral oil should not mix with the sample. Pipette tips, containing samples, should be inserted firmly in the inlets of the microfluidic device, with utmost precaution, to prevent leakage and further incorporation of air bubbles. To summarize, tip-loading is a straightforward, yet, robust technique that allows for high-throughput analysis of cellular behavior through cell encapsulation without significant loss of cells in a cost-effective manner. When used with optimal sample concentrations at the inlet, this approach of loading cells with pipette-tips is very flexible and can be adapted for different cell types, especially for rare primary immune cells, to obtain higher encapsulation efficiency, close to predicted models.

\section{Disclosures}

We have nothing to disclose.

\section{Acknowledgments}

We thank the Eindhoven University of Technology for generous support.

\section{References}

1. Yin, H., \& Marshall, D. Microfluidics for single cell analysis. Current Opinion in Biotechnology. 23 (1), 110-119 (2012).

2. Meyvantsson, I., \& Beebe, D. J. Cell Culture Models in Microfluidic Systems. Annual Review of Analytical Chemistry. 1, 423-429 (2008).

3. Yi, C., Li, C. W., Ji, S., \& Yang, M. Microfluidics technology for manipulation and analysis of biological cells. Analytica Chimica Acta. 560, 1-23 (2006).

4. Wang, H. Y., Bao, N., \& Lu, C. A microfluidic cell array with individually addressable culture chambers. Biosensors and Bioelectronics. 24, 613-617 (2008).

5. Zhang, Y., et al. A programmable microenvironment for cellular studies via. microfluidics-generated double emulsions. Biomaterials. 34 (19), 4564-4572 (2013).

6. Teh, S.-Y., Lin, R., Hung, L.-H., \& Lee, A. P. Droplet microfluidics. Lab on a chip. 8 (2), 198-220 (2008).

7. Hu, H., et al. Efficient cell pairing in droplets using dual-color sorting. Lab Chip. 15 (20), 3989-3993 (2015).

8. Brouzes, E., et al. Droplet microfluidic technology for single-cell high-throughput screening. Proceedings of the National Academy of Sciences. 106 (34), 14195-14200 (2009).

9. Chokkalingam, V., et al. Probing cellular heterogeneity in cytokine-secreting immune cells using droplet-based microfluidics. Lab on a Chip. 13 (42), 4740 (2013).

10. den Haan, J. M. M., Arens, R., \& van Zelm, M. C. The activation of the adaptive immune system: Cross-talk between antigen-presenting cells, T cells and B cells. Immunology Letters. 162 (2), 103-112 (2014).

11. Shah, G. J., Ohta, A. T., Chiou, E. P.-Y., Wu, M. C., \& Kim, C.-J. EWOD-driven droplet microfluidic device integrated with optoelectronic tweezers as an automated platform for cellular isolation and analysis. Lab on a Chip. 9 (12), 1732 (2009).

12. Griffiths, A. D., \& Tawfik, D. S. Miniaturising the laboratory in emulsion droplets. Trends in Biotechnology. 24 (9), $395-402$ (2006).

13. Lagus, T. P., \& Edd, J. F. High-throughput co-encapsulation of self-ordered cell trains: cell pair interactions in microdroplets. RSC Advances. 3, (43), 20512 (2013).

14. Moon, S., Ceyhan, E., Gurkan, U. A., \& Demirci, U. Statistical modeling of single target cell encapsulation. PLoS ONE. 6 (7), (2011). 
15. Abate, A. R., Chen, C.-H., Agresti, J. J., \& Weitz, D. A. Beating Poisson encapsulation statistics using close-packed ordering. Lab on a Chip. 9 (18), 2628 (2009).

16. Collins, D. J., Neild, A., deMello, A., Liu, A.-Q., \& Ai, Y. The Poisson distribution and beyond: methods for microfluidic droplet production and single cell encapsulation. Lab Chip. 15 (17), 3439-3459 (2015).

17. Kemna, E. W. M., et al. High-yield cell ordering and deterministic cell-in-droplet encapsulation using Dean flow in a curved microchannel. Lab on a Chip. 12 (16), 2881 (2012).

18. Köster, S., et al. Drop-based microfluidic devices for encapsulation of single cells. Lab on a Chip. 8 (7), 1110 (2008).

19. Mazutis, L., et al. Single-cell analysis and sorting using droplet-based microfluidics. Nature Protocols. 8 (5), $870-891$ (2013).

20. Sun, P., et al. Functional characterization of ex vivo. blood myeloid and plasmacytoid dendritic cells after infection with dengue virus. Virology. 383 (2), 207-215 (2009).

21. Tel, J., et al. The chemotherapeutic drug oxaliplatin differentially affects blood DC function dependent on environmental cues. Cancer Immunology, Immunotherapy. 61 (7), 1101-1111 (2012).

22. Wimmers, F., et al. Single-cell analysis reveals that stochasticity and paracrine signaling control interferon-alpha production by plasmacytoid dendritic cells. Nature Communications. 9 (1), 3317 (2018).

23. Gong, J., \& Kim, C.-J. All-electronic droplet generation on-chip with real-time feedback control for EWOD digital microfluidics. Lab on a Chip. 8 (6), 898 (2008).

24. Demirci, U., \& Montesano, G. Single cell epitaxy by acoustic picolitre droplets. Lab on a Chip. 7 (9), 1139 (2007).

25. Rho, H. S., Yang, Y., Veltkamp, H.-Willem \& Gardeniers, H. Direct Delivery of Reagents from a Pipette Tip to a PDMS Microfluidic Device. Chips and Tips. at <http://blogs.rsc.org/chipsandtips/2015/10/09/direct-delivery-of-reagents-from-a-pipette-tip-to-a-pdms-microfluidic-device/> (2015).

26. Paul, P. H., Garguilo, M. G., \& Rakestraw, D. J. Imaging of Pressure- And Electrokinetically Driven Flows through Open Capillaries. Analytical Chemistry. 70 (13), 2459-2467 (1998).

27. Whitesides, G. M., \& Stroock, A. D. Flexible methods for microfluidics. Physics Today. 54, 42 (2001).

28. Mita, A., et al. Anti-proinflammatory Effects of lodixanol (OptiPrep)-Based Density Gradient Purification on Human Islet Preparations. Cell Transplant. 19 (12), 1537-1546 (2013).

29. Dura, B., et al. Profiling lymphocyte interactions at the single-cell level by microfluidic cell pairing. Nature Communications. 6 (1), 1-13 (2015).

30. Dura, B., et al. Longitudinal multiparameter assay of lymphocyte interactions from onset by microfluidic cell pairing and culture. Proceedings of the National Academy of Sciences. 113 (26), E3599-E3608 (2016).

31. Yamanaka, Y. J., et al. Single-cell analysis of the dynamics and functional outcomes of interactions between human natural killer cells and target cells. Integrative Biology. 4 (10), 1175 (2012).

32. Satija, R., \& Shalek, A. K. Heterogeneity in immune responses: From populations to single cells. Trends in Immunology. 35 (5), $219-229$ (2014). 